11

\title{
Рост кристаллов при спонтанной кристаллизации в неинерциальных системах в условиях космической станции и в условиях Земли на примере синтеза и роста кристаллов $\mathrm{CrSi}_{2}$ из раствора-расплава Zn
}

\author{
(C) Е.В. Калашников ${ }^{1,2}$, В.Н. Гурин ${ }^{2}$, С.П. Никаноров ${ }^{2}$, Л.И. Деркаченко ${ }^{2}$, М.А. Яговкина \\ ${ }^{1}$ Московский государственный областной университет, \\ Мытищи, Московская обл., Россия \\ ${ }^{2}$ Физико-технический институт им. А.Ф. Иофрфе РАН, \\ Санкт-Петербург, Россия \\ E-mail: ekevkalashnikov1@gmail.com
}

Поступила в Редакцию 16 июля 2019 г.

В окончательной редакции 16 июля 2019 г.

Принята к публикации 25 июля 2019 г.

\begin{abstract}
Рассматривается влияние гравитационного поля Земли на рост кристаллов из раствора-расплава при спонтанной кристаллизации. Для этого принимается во внимание, что космическая станция (КС) и лаборатория на Земле, в которых протекают процессы кристаллизации, являются неинерциальными системами. Показано, что особенностью отличающей рост кристалла на Земле является давление в расплаве, вызванное реакцией опоры (третий закон Ньютона). Такое давление на КС отсутствует и это ведет к увеличению элементарного объема расплава, претерпевающего фазовый переход первого рода. В результате кристаллы на КС оказываются по размерам бо́льшими, чем те же кристаллы, выращенные на Земле. И они обладают избыточным напряжением, по величине равным отсутствующему на КС давлению опоры. Эта ситуация сопоставляется с экспериментальными данными по выращиванию кристаллов $\mathrm{CrSi}_{2}$ из раствора-расплава в $\mathrm{Zn}$ системы $\mathrm{Cr}-\mathrm{Si}-\mathrm{Zn}$.
\end{abstract}

Ключевые слова: спонтанная кристаллизация, расплав-раствор, неинерциальная система, реакция опоры, невесомость, несмачиваемость, химический потенциал.

DOI: $10.21883 /$ FTT.2020.01.48728.52ks

\section{1. Введение}

Эксперименты показывают, что кристаллизация в условиях невесомости (космической станции, КС) и в условиях Земли значительно отличаются [1-8]. В частности отмечается существенное влияние условий невесомости [4] на форму выращенных кристаллов на форму кристаллов таких как $\mathrm{CrSi}_{2}[1,2],{ }^{4} \mathrm{He}$ [5] и $\mathrm{InGaSb}$ [7].

Причиной такого различия являются особенности, связанные с доставкой вещества к растущему кристаллу. Одной из возможных таких причин для синтеза и спонтанной кристаллизации (массовой кристаллизации) на КС (невесомость) является отсутствие конвективного движения в жидкости [9], вызванного неоднородным распределением температуры (градиент температуры) [10]. Но здесь будет массоперенос, вызванный градиентом химического потенциала, который определяется „разностью“ одного и того же вещества в разных точках расплава. Температура и состав расплава являются хорошо контролируемыми параметрами. Однако, зависимость химического потенциала от давления в условиях Земли и КС неочевидна. Также неочевидно, что означает давление в расплаве в случае зарождения кристаллов и их роста.

Важные результаты экспериментов по синтезу и появлению кристаллов $\mathrm{CrSi}_{2}$ при спонтанной кристал- лизации в растворе-расплаве в системы [1] показали следующее. Микрокристаллы, выращенные в условиях невесомости (a) имеют изометрическую форму (размеры кристаллов вдоль трех направлений приблизительно равны) $\sim 600 \mu \mathrm{m}$. Эти кристаллы в $1.5-2$ раза превышают размеры кристаллов того же соединения, выращенного в условиях Земли $(\sim 300 \mu \mathrm{m})$; (b) имеют увеличенное число граней; $(c)$ по составу отличаются от стехиометрического; $(d)$ микротвердость в условиях невесомости $-H_{V}=1200 \pm 150$, а в условиях Земли $H_{N}=1450 \pm 150 \mathrm{GPa}$.

Эти факты, в частности, $(a)$ и $(d)$, указывают на два очень важных свойства кристаллов, выращенных в условиях невесомости. Кристаллы, выросшие в условиях невесомости и помещенные в условия Земли (i) оказываются в напряженном состоянии, (ii) имеют больше дефектов (чем кристаллы, выросшие в условиях Земли).

Влияние „весомости“ и „невесомости“ на процесс образования и рост кристаллов не вполне ясен. Земля и Космическая станция, в общем, являются неинерциальными системами. Поэтому необходимо уточнить понятие явления весомости и невесомости и как эти явления проявляют себя в условиях роста кристаллов. В обоих случаях (Земли и КС) капсулы с расплавом, в котором возникают и растут кристаллы, находятся, в 
общем, в неинерциальных системах отсчета. Поэтому необходимо учесть поведение вещества в таких системах отсчета.

\section{2. Влияние неинерциальной системы отсчета на условия кристаллизации}

\section{1. Общий случай}

Космическая станция (КС) вращается вокруг Земли с постоянной угловой скоростью $\omega$ на расстоянии $\left|\mathbf{r}_{\mathrm{ES}}\right|$ от центра Земли до КС и является неинерциальной системой. Уравнение движения центра масс расплава в капсуле, закрепленной на КС следующее $[11,12]$ :

$$
\begin{aligned}
m\left(d^{2} \mathbf{r}_{c} / d t^{2}\right)=\mathbf{R}+\mathbf{F} & -m\left(d^{2} \mathbf{r}_{\mathrm{ES}} / d t^{2}\right) \\
& -m\left[\boldsymbol{\omega}\left[\boldsymbol{\omega} \mathbf{r}_{c}\right]\right]-2 m\left[\boldsymbol{\omega} \mathbf{v}_{c}\right] .
\end{aligned}
$$

Здесь $\mathbf{r}_{c}$ - радиус-вектор центра масс расплава в капсуле относительно системы отсчета, связанной с КС; $\mathbf{R}$ - реакция опоры, сила, которую нужно найти, она действует при фиксации капсулы внутри $\mathrm{KC} ; \mathbf{F}-$ сила притяжения центра масс расплава в капсуле к Земле

$$
\mathbf{F}=-\gamma\left(\mathbf{M}_{\mathrm{E}} m / \mathbf{r}_{a}^{3}\right) \mathbf{r}_{a},
$$

$\gamma$ - гравитационная постоянная, $\mathrm{M}_{\mathrm{E}}$ - масса Земли, $m$ - центр масс расплава, $\mathbf{r}_{a}$ - расстояние от центра Земли до центра масс расплава; $\mathbf{r}_{\mathrm{ES}}$ - центр масс КС; $m_{j}$ и $\mathbf{r}_{j}-$ являются $j$-ми частями массы КС и радиус-векторами этих частей относительно Земли, соответственно. Это значит, что

$$
\mathbf{r}_{\mathrm{ES}}=\Sigma_{j} m_{j} \mathbf{r}_{j} / \Sigma_{j} m_{j}
$$

Но на КС действует сила притяжения Земли

$$
m_{\mathrm{SS}}\left(d^{2} \mathbf{r}_{\mathrm{ES}} / d t^{2}\right)=-\gamma \Sigma_{j}\left(\mathrm{M}_{\mathrm{E}} m_{j} / r_{j}^{3}\right) \mathbf{r}_{j} .
$$

Отметим, что $\Sigma_{j} m_{j} \mathbf{r}_{j}=m_{\mathrm{SS}} \mathbf{r}_{\mathrm{ES}}$ (где $\Sigma_{j} m_{j}=m_{\mathrm{SS}}$ ), и $\left|\mathbf{r}_{j}-\mathbf{r}_{j+1}\right| \ll \mathbf{r}_{\mathrm{ES}}$ и $\mathbf{r}_{j} \approx \mathbf{r}_{\mathrm{ES}}$, тогда (1) при условии $r_{a} \cong \mathbf{r}_{\mathrm{ES}}$ сводится к

$$
m\left(d^{2} \mathbf{r}_{c} / d t^{2}\right)=\mathbf{R}-m\left[\boldsymbol{\omega}\left[\boldsymbol{\omega} \mathbf{r}_{c}\right]\right]-2 m\left[\boldsymbol{\omega} \mathbf{v}_{c}\right] .
$$

Теперь учтем, что капсула с расплавом зафиксирована внутри КС. Тогда движение капсулы с расплавом в КС отсутствует и $\left[\omega \mathbf{v}_{c}\right]=0$, и $\mathbf{v}_{c}=0$ и $d^{2} \mathbf{r}_{c} / d t^{2}=0$. В этом случае выражение (5) превращается: $\mathbf{R}-m\left[\boldsymbol{\omega}\left[\boldsymbol{\omega} \mathbf{r}_{c}\right]\right]=0$. А вес капсулы в КС вращающейся вокруг Земли с угловой скоростью $\omega$, равен

$$
\mathbf{R}=m\left[\boldsymbol{\omega}\left[\omega \mathbf{r}_{c}\right]\right],
$$

величина которой $R=m \omega^{2} r_{c}=5.33 \cdot 10^{-5} N$. Если $\mathbf{r}_{c}$ капсулы ориентирован параллельно оси вращения КС относительно Земли, т.е. $\boldsymbol{\omega} \| \mathbf{r}_{c}$, тогда из (6) $\mathbf{R}=0$ и капсула с расплавом находится в полной невесомости.

\section{2. На Земле}

Если теперь КС закреплена на Земле, тогда уравнение (1) принимает иную форму. Теперь $\omega_{\mathrm{E}}$ равна угловой скорости поверхности Земли относительно ее собственной оси (вместо $\omega$ ) и $r_{a}=R_{\mathrm{E}}=6.4 \cdot 10^{6} \mathrm{~m}-$ радиус Земли. Уравнение (1) теперь превращается в уравнение движения расплава в капсуле скрепленной с Землей и вращающейся вместе с Землей относительно ее центра: $0=\mathbf{R}+\mathbf{F}-m\left[\boldsymbol{\omega}_{\mathrm{E}}\left[\boldsymbol{\omega}_{\mathrm{E}} \mathbf{r}_{c}\right]\right]$ или

$$
\mathbf{R}=\gamma\left(\mathbf{M}_{\mathrm{E}} m / r_{a}^{3}\right) \mathbf{r}_{a}+m\left[\omega_{\mathrm{E}}\left[\omega_{\mathrm{E}} \mathbf{r}_{c}\right]\right] .
$$

Для оценки значений величин реакции опоры использовались следующие данные estimate угловая скорость вращения Земли $\omega_{\mathrm{E}} \approx 7.3 \cdot 10^{-5} \mathrm{~s}^{-1}$, масса Земли $\mathrm{M}_{\mathrm{E}}=5.9736 \cdot 10^{24} \mathrm{~kg}$; гравитационная постоянная: $\gamma=6.67259 \cdot 10^{-11} \mathrm{~m}^{3} / \mathrm{kg} \mathrm{s}^{2}$; высота орбиты КС над Землей $\approx 400 \cdot 10^{3} \mathrm{~m}$; угловая скорость обращения КС относительно Земли $\omega \approx 7.3 \cdot 10^{-4} \mathrm{~s}^{-1}$. Тогда

$$
|\mathbf{R}| \approx 9.7 \cdot 10^{-2} \mathrm{~N} .
$$

Это значит, что сила реакции опоры на Земле больше чем та же реакция опоры на КС в $1.82 \cdot 10^{3}$ раза, при учете (6). Теперь оценим давление, вызванное реакцией опоры. Рассмотрим давление в расплаве.

\section{3. Экспериментальная ситуации, в которой возникают микрокристаллы $\mathrm{CrSi}_{2}$}

Одинаково приготовленные образцы $[1,2]$ размещались в цинковой капсуле в кварцевую цилиндрическую ампулу. Ампулы откачивалась и запаивалась. При температурах плавления и выше наступает значительное различие в поведении расплава на Земле и на КС.

\section{1. Условия на Земле}

Внешнее давление действует на раствор-расплав цинка $P_{\text {ext. }}$ Оно вызвано давлением насыщенного пара в замкнутой ампуле. К нему добавляется давление Лапласа, вызванное несмачиваемостью (знак „,+“) или смачиваемостью (знак „-““) расплава внутренних стенок кварцевой ампулы $\pm \Delta P_{\text {Lapl. }}$. на КС) возникает, в соответствии с (7) и (7a), давление вызванное реакцией опоры $P_{\text {sup. }}$. нее давление $P_{\text {int }}$ в растворе-расплаве цинка изменится

$$
P_{\text {int }} \rightarrow P_{\text {int }}+P_{\text {ext }} \pm \Delta P_{\text {Lapl }}+P_{\text {sup }} .
$$

\section{2. Условия на $\mathrm{KC}$}

Внутренне давление в растворе-расплаве цинка, согласно (6) и (7), будет выглядеть

$$
P_{\text {int }} \rightarrow P_{\text {int }}+P_{\text {ext }} \pm 2 \Delta P_{\text {Lapl }} .
$$

Для цинка при температуре плавления $T=723 \mathrm{~K}$, $\rho=6.6 \cdot 10^{3} \mathrm{~kg} / \mathrm{m}^{3} ; \gamma=755 \cdot 10^{-3} \mathrm{~J} / \mathrm{m}^{2}$, для диаметра 
кварцевой ампулы $2 r=1 \cdot 10^{-2} \mathrm{~m}$ и высотой расплава $3 \cdot 10^{-2} \mathrm{~m}$, давление $P_{\text {sup }}=1940 \mathrm{~Pa}$ превышает $\Delta P_{\text {Lapl }}=151 \mathrm{~Pa}$ на порядок. Однако, при плавлении и более высоких температурах происходит растворение и смешивание компонентов. Это ведет к тому, что поверхностное натяжение будет меняться [13], и смачиваемость может меняться на несмачиваемость, и наоборот. Но $P_{\text {sup }}$ больше $\Delta P_{\text {Lapl }}$ и потому влияние $\Delta P_{\text {Lapl }}$ будет влиять на расплав значительно слабее. Но кривизна границы раздела будет влиять на работу образования кристалла в расплаве.

\section{4. Определение состояния кристалла, выращенного в КС}

В задачах роста кристаллов, контролирующими параметрами обычно являются температура и состав. Удобным термодинамическим потенциалом для описания системы, состоящей из одного вещества, в котором растет кристалл является потенциал Гиббса $G=U+P V=T S$. Учитывая его аддитивность, можно ввести химический потенциал каждого вида вещества

$$
G_{1}=n(u+P v-T s)_{j}=n \mu_{j}(P, T) .
$$

Здесь $n-$ число атомов(молекул) определенного типа вещества, $j-$ соответствует жидкому (L) или кристаллическому $(\mathrm{Cr})$ состоянию.

\section{1. Определение избыточного напряжения кристалла}

Возникновение кристалла является процессом фазового перехода первого рода и носит локальный характер. Это означает, что только часть жидкого расплава объема $V_{\mathrm{L}}$ претерпевает превращение в кристалл и соответственно число $(n)$ атомов(молекул) в этом объеме полностью трансформируется в кристалл объема $V_{\text {cr }}$. Из (10) имеем

$$
\left(\partial \mu_{j}(P, T) / \partial P\right)_{T}=v_{j}=V_{j} / n, \quad j=\mathrm{L}, \mathrm{Cr} .
$$

Интегрируя (11), получаем $n \cdot \mu_{j}=\int_{p_{\mathrm{L}-\mathrm{cr}}}^{p} V_{j} d P \quad$ [14]. Сравним эти два $(j=\mathrm{L}, \mathrm{Cr})$ выражения. (В равновесии $\mu_{\mathrm{L}}=\mu_{\mathrm{cr}}$, тогда $P-P_{\mathrm{L}-\mathrm{cr}}=0$, здесь $P_{\mathrm{L}-\mathrm{cr}}$ - давление на границе „расплав-кристалл“ в равновесии). Поэтому рассматриваем условия, при которых существует движение границы раздела. А это, кстати, задает кинетику роста кристалла, при которой кристалл растет и приобретает грани

$$
\begin{aligned}
n\left(\mu_{\mathrm{L}}-\mu_{\mathrm{cr}}\right) & =\int_{P_{\mathrm{L}-\mathrm{cr}}}^{p}\left(V_{\mathrm{L}}-V_{\mathrm{cr}}\right) d P \\
& =\left(P-P_{\mathrm{L}-\mathrm{cr}}\right)\left(V_{\mathrm{L}}-V_{\mathrm{cr}}\right)
\end{aligned}
$$

или

$$
n\left(\mu_{\mathrm{L}}-\mu_{\mathrm{cr}}\right)=\left(P-P_{\mathrm{L}-\mathrm{cr}}\right)\left(V_{\mathrm{L}}-V_{\mathrm{cr}}\right)
$$

при $T=$ const (11). Для отслеживания того, как и в чем разница в росте кристалла в условиях Земли и КС используются соотношения (12). Для условий на Земле

$$
n\left(\mu_{\mathrm{L}}-\mu_{\mathrm{cr}}\right)_{\mathrm{E}}=\left(P-P_{\mathrm{L}-\mathrm{cr}}\right)_{\mathrm{E}}\left(V_{\mathrm{L}}-V_{\mathrm{cr}}\right)_{\mathrm{E}}
$$

Аналогичное уравнение для КС

$$
n\left(\mu_{\mathrm{L}}-\mu_{\mathrm{cr}}\right)_{\mathrm{SS}}=\left(P-P_{\mathrm{L}-\mathrm{cr}}\right)_{\mathrm{SS}}\left(V_{\mathrm{L}}-V_{\mathrm{cr}}\right)_{\mathrm{SS}} .
$$

Левые части последних двух уравнений (13) и (13a) равны, поскольку они определяются только работой по переносу n атомов (молекул) из расплава к кристаллу. Тогда и правые части этих уравнений равны

$$
\left(P-P_{\mathrm{L}-\mathrm{cr}}\right)_{\mathrm{E}}\left(V_{\mathrm{L}}-V_{\mathrm{cr}}\right)_{\mathrm{E}}=\left(P-P_{\mathrm{L}-\mathrm{cr}}\right)_{\mathrm{SS}}\left(V_{\mathrm{L}}-V_{\mathrm{cr}}\right)_{\mathrm{SS}}
$$

Разность $\left(P-P_{\mathrm{L}-\mathrm{cr}}\right)_{\mathrm{E}}$ отличается от $\left(P-P_{\mathrm{L}-\mathrm{cr}}\right)_{\mathrm{SS}}$ на величину $P_{\text {sup }}$ (здесь давлением Лапласа пренебрегаем). Тогда

$$
\left(P-P_{\mathrm{L}-\mathrm{cr}}\right)_{\mathrm{SS}}=\left(P-P_{\mathrm{L}-\mathrm{cr}}\right)_{\mathrm{E}}-P_{\text {sup }}
$$

а уравнение (14) теперь приобретает вид

$$
\begin{aligned}
&\left(P-P_{\mathrm{L}-\mathrm{cr}}\right)_{\mathrm{E}}\left(V_{\mathrm{L}}-V_{\mathrm{cr}}\right)_{\mathrm{E}} \\
&=\left(\left(P-P_{\mathrm{L}-\mathrm{cr}}\right)_{\mathrm{E}}-P_{\text {sup }}\right)\left(V_{\mathrm{L}}-V_{\mathrm{cr}}\right)_{\mathrm{SS}}
\end{aligned}
$$

Учтем, что размер кристалла выросшего на КС в 1.5-2 раза больше чем на Земле (экспериментальный результат $(a)$ и $[1])$ :

$$
\left(V_{\mathrm{L}}-V_{\mathrm{cr}}\right)_{\mathrm{SS}}=2\left(V_{\mathrm{L}}-V_{\mathrm{cr}}\right)_{\mathrm{E}}
$$

т.е. кристалл не может вырасти больше, если ему не предоставлен бо́льший объем. Возвращаясь к (15) получаем:

$$
\begin{aligned}
\left(P-P_{\mathrm{L}-\mathrm{cr}}\right)_{\mathrm{E}}\left(\left(V_{\mathrm{L}}-V_{\mathrm{cr}}\right)_{\mathrm{E}}\right. & \left.-2\left(V_{\mathrm{L}}-V_{\mathrm{cr}}\right)_{\mathrm{E}}\right) \\
= & \left(-P_{\text {sup }}\right) 2\left(V_{\mathrm{L}}-V_{\mathrm{cr}}\right)_{\mathrm{E}}
\end{aligned}
$$

или

$$
2 P_{\text {sup }}=\left(P-P_{\mathrm{L}-\mathrm{cr}}\right)_{\mathrm{E}} \text {. }
$$

Подставляя это выражение в (14a), получаем

$$
\left(P-P_{\mathrm{L}-\mathrm{cr}}\right)_{\mathrm{SS}}=2 P_{\text {sup }}-P_{\text {sup }}=P_{\text {sup }}
$$

Это означает, что кристалл, выросший на КС, имеет избыточное напряжение

$$
\sigma_{j k}=\delta_{j k} P_{\text {sup }}
$$

Модуль которого равен $\left|\sigma_{j k}\right|=P_{\text {sup. }}$ 


\section{2. Огранки кристалла}

Другая важная особенность связана с формированием граней кристалла. В соответствии с Вольфом [14], кристалл может поделен на пирамиды, вершины которых начинаются из некоторой внутренней точки кристалла О. Тогда объем кристалла равен сумме объемов пирамид:

$$
\left(V_{\mathrm{L}}-V_{\mathrm{cr}}\right)_{\mathrm{E}}=\sum_{j}(1 / 3) A_{j} h_{j} .
$$

Здесь $\left(V_{\mathrm{L}}-V_{\mathrm{cr}}\right)_{\mathrm{E}}$ - объем выросшего кристалла на Земле, $A_{j}$ - площадь $j$-й грани, $h_{j}-$ расстояние от центра кристалла О до $j$-й грани. Но поскольку условия невесомости обеспечивают бо́льший объем (16), то и число граней окажется бо́льшим.

\section{5. Заключение}

Показано, что при отсутствии давления, вызванного реакцией опоры, в расплаве, в космической станции микрокристаллы увеличиваются в размерах. Они имеют избыточное напряжение, равное по величине давлению в расплаве вызванного реакцией опоры в условиях Земли.

\section{Финансирование работы}

Работа выполнена при частичной финансовой поддержке Министерства образования и науки РФ (идентификатор проекта RFMEFI62117X0018) в рамках исследований по теме 9.7 (0040-2014-0007).

\section{Конфликт интересов}

Авторы заявляют, что у них нет конфликта интересов.

\section{Список литературы}

[1] V.N. Gurin, L.I. Derkatchenko, S.P. Nikanorov. Proc. AIAA/IKI Microgravity Science Symposium (13-17 May 1991, Moscow) Pabl. AIAA, Washington (1991). P. 134.

[2] В.И. Стрелов, И.П. Куранова, Б.Г. Захаров, А.Э. Волошин. Кристаллография 59, 863 (2014).

[3] K. Zhang, L.B. Wang. Frontiers Heat Mass Transfer 7, 1 (2016).

[4] C.E. Kundrot, R.A. Judge, M.L. Pusey, E.H. Snell. Cryst. Growth Design 1, 87 (2001).

[5] T. Takuya, O. Haruka, N. Ryuji, O. Yuichi. Sci. Adv. 1:e1500825, 1 (2015).

[6] N.S. Vonortas. Protein crystallization for drug development: Final Rep. Nasa (2015). 30 p.

[7] Y. Hayakawa, V.N. Kumar, M. Arivanandhan, G. Rajesh, T. Koyama, Y. Momose, K. Sakata, T. Ozawa, Y. Okano, Y. Inatomi. J. Microgravity Sci. Appl. 34, 340111 (2017).

[8] И.Л. Шульпина, Б.Г. Захаров, Р.В. Парфеньев, И.И. Фарбштейн, Ю.А. Серебряков, И.А. Прохоров. ФТТ 54, 1264 (2012).

[9] П.К. Волков. Природа 11, 36 (2001).
[10] Гидромеханика и тепломассообмен в невесомости / Под ред. В.С. Авдуевского, В.И. Полежаева. Наука, М. (1982). $263 \mathrm{c}$.

[11] Л.Д. Ландау, Е.М. Лифшиц. Механика. Наука, М. (1988). $215 \mathrm{c}$.

[12] И.И. Ольшанский. Курс теоретической механики для физиков. МГУ, М. (1974). 569 с.

[13] А.Г. Амброк, Е.В. Калашников. Расплавы 4, 41 (1997).

[14] М. Фольмер. Кинетика образования новой фазы. Наука, М. (1986). 208 c.

Редактор Т.Н. Василевская 